\title{
Design, Synthesis, Antimicrobial and Anti-inflammatory Activity of N-Pyrazolyl Benzamide Derivatives
}

Aneesa Fatima ${ }^{1 *}$, Ravindra Kulkarni ${ }^{2}$ and Bhagavanraju Mantipragada ${ }^{3}$

${ }^{1}$ Malla Reddy College of Pharmacy, Maisammaguda, Secunderabad, Telangana, India

${ }^{2}$ SVERI College of Pharmacy, Gopalpur Ranjini Road, Gopalpur, Pandharpur, Maharashtra, India

${ }^{3}$ Sri Venkateswara College of Pharmacy, HITEC City, Madhapur, Telangana, India

\begin{abstract}
New N-Pyrazolylbenzamide derivatives which possess 2, 4-dinitrophenyl group were synthesized using 4, 4-dimethyl-3-oxo-pentanenitrile and 2, 4-dinitrophenyl hydrazine hydrochloride to afford an intermediate compound. The intermediate on auxiliary aroylation with substituted benzoyl chlorides in the presence of base yielded the subsequent N-Pyrazolylbenzamide derivatives (5a-l). The structures of newly synthesized compounds were elucidated by ${ }^{1} \mathrm{H}$ NMR, FT IR and Mass spectral analysis. The anti-inflammatory activity of all newly synthesized was evaluated using carrageenan induced paw edema model and antimicrobial activity by serial dilution method. Six compounds ( $5 \mathrm{~d}$, $5 \mathrm{f}$ and $5 \mathrm{~h}-\mathrm{k}$ ) showed consistently good anti-inflammatory activity in particular 4-trifluoromethyl-. N-[3'-t-butyl-1'-(2", 4"-dinitro)phenylpyrazol-5'-yl] benzamide (5h) was found to be the most effective among the other derivatives. The antimicrobial screening of all synthesized molecules showed that compounds $5 \mathrm{f}$ and $5 \mathrm{~h}$ possess superior and encouraging activity against tested organisms.
\end{abstract}

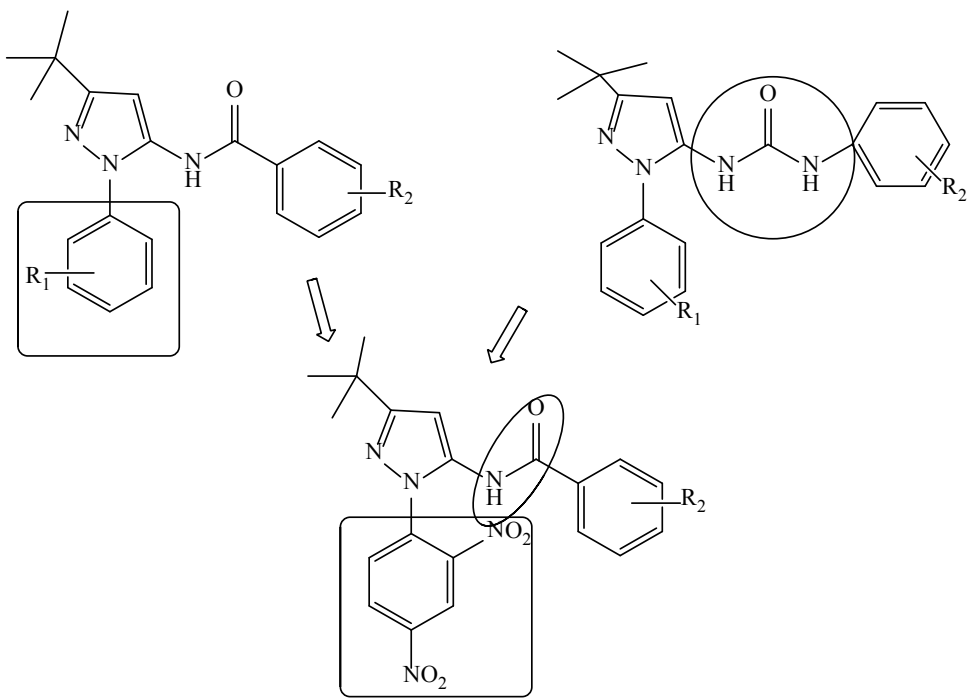

New N-Pyrazolylbenzamide derivatives containing 2, 4-dinitrophenyl group were designed from diarylureas which resulted in $\mathrm{N}$-(3-tert-butyl-2, 4-dinitro-1-phenyl-1H-pyrazol-5-yl)benzamide derivatives. All the compounds were screened for antimicrobial and anti-inflammatory activity.

Keywords: Pyrazole; Benzamides; Anti-inflammatory; Antibacterial; Antifungal

Abbreviations: DMSO: Dimethyl sulfoxide; h: Hour; s: Singlet; d: Doublet; t: Triplet; bs: Broad singlet; m: Multiplet; IR: Infrared; ${ }^{1} \mathrm{H}$ NMR: Proton nuclear magnetic resonance; Mol: Moles; $\mathrm{M}$. Wt.: Molecular weight; mL: Milli liters; m.p.: Melting point; min: Minutes; mm: Millimetres; ${ }^{\circ} \mathrm{C}$ : Degree Centigrade; $\mathrm{CDCl}_{3}$ : Chloroform (Deuteriated); DCM: Dichloromethane; TLC: Thin layer chromatography; str: Stretching; Bnd: Bending; spp: Species; $\mu$ g: Micro gram

\section{Introduction}

Inflammation is a key area of research for many pharmaceutical companies. Patients suffering from inflammatory disorders including rheumatoid arthritis (RA) require therapeutic agents that not only to demonstrate anti-inflammatory properties but also to protect against cartilage degradation [1]. TNF- $\alpha$ and IL- $1 \beta$ which are also known as proinflammatory cytokines are frequently found in numerous inflammatory diseases at elevated levels [2,3]. Clinically to alleviate inflammation steroidal and nonsteroidal anti-inflammatory agents are employed [4] and maximum of such agents have shown severe side effects which aspire need of safe antiinflammatory agents [5].

Twentieth century is known for its contribution in medical

*Corresponding author: Aneesa Fatima, Malla Reddy College of Pharmacy Maisammaguda, Secunderabad, Telangana, India, Tel: +918125343156; E-mail: aneesafatima.16@gmail.com

Received November 17, 2015; Accepted December 07, 2015; Published December 10, 2015

Citation: Fatima A, Kulkarni R, Mantipragada B (2015) Design, Synthesis, Antimicrobial and Anti-inflammatory Activity of N-Pyrazolyl Benzamide Derivatives. Med chem 5: 521-527. doi: 10.4172/2161-0444.1000311

Copyright: ( 2015 Fatima A, et al. This is an open-access article distributed under the terms of the Creative Commons Attribution License, which permits unrestricted use, distribution, and reproduction in any medium, provided the original author and source are credited. 
fraternity for controlling various microbial diseases including tuberculosis [6]. Many diseases which were caused by microbes were effectively contained and this was possible because of search of new and potent antimicrobial agents either from natural sources or synthetic routes [7-9]. Last decade of $20^{\text {th }}$ century and early part of $21^{\text {st }}$ century is contradictory to the remarkable success of medical sciences for the treatment of infectious diseases. This is mainly due to emergence of resistant strains of organisms which resulted in ineffectiveness of many antibiotics in the clinical treatment and also lackluster approach of giant pharmaceutical company towards new antimicrobial drug research [10-12]. Most of the available antibacterial agents have now become least effective or ineffective against methicillin resistant Staphylococcus aureus, vancomycin resistant Staphylococcus aureus, resistant strains of Escherichia coli and new bacteria which express genes encoded for carbepenamase etc. [13]. Apart from this, not a single antibiotic with different chemical structure and mechanism of action has reached to clinical stage. Thus infectious diseases are still known to be the cause of mortality among the third world countries including India and the need of potent and safe clinical agents to contain infectious disease still persists [14].

Pyrazole and its derivatives occupy an important position in medicinal and pesticide chemistry due to a wide range of bioactivities such as antimicrobial [15], anticancer [16], anti-inflammatory [17], antidepressant, anticonvulsant, antihyperglycemic, antipyretic, antibacterial, antifungal, antiviral [18] and selective enzyme inhibitory activities [19]. Substituted pyrazole and its analogs have been used as precursors for synthesis of various biologically active molecules [20].

Our group is extensively working on 5-aminopyrazole derivatives as antiinflammatory agent and are first to report that pyrazole compounds with a tertiary butyl groups also demonstrate important antimicrobial activity [21]. In the previous publication, a simple monosubstituted aromatic group was inserted at the $1^{\text {st }}$ position of pyrazole and one such molecule VHw (Figure 1) exhibited antibacterial activity in nano gram $/ \mathrm{mL}$ range which encouraged us to explore chemical space in the substitutions on pyrazole scaffold and synthesize new generation with disubstituted aromatic group at the same position. This manuscript is aimed to report the synthesis and pharmacological activities of pyrazole containing benzamide compounds bearing a 2, 4-dinitrophenyl group at the $1^{\text {st }}$ position of pyrazole.

\section{Materials and Methods}

Six stage reaction station Radleys discovery Technologies,

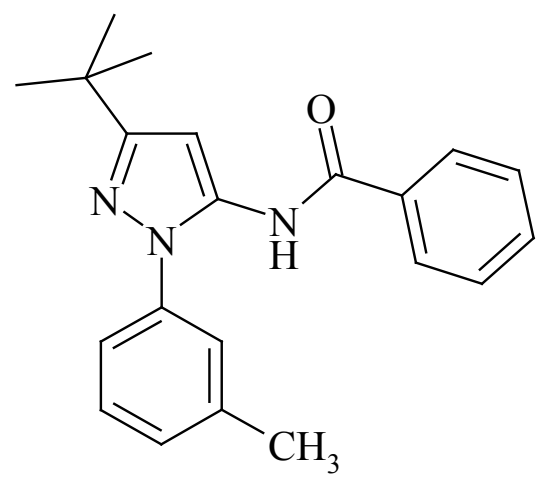

VHw, $400 \mathrm{ng} / \mathrm{mL}$

Figure 1: Structure of $\mathrm{VHw}$ along with antibacterial activity against Bacillus subtilis (MTCC 619).
Germany was used for important reactions. The course of reaction and purity were ascertained by performing TLC. Chemicals and solvents were obtained from S. D. Fine chemicals, Merck and Sigma Aldrich; before use solvents were purified. The melting points of the synthesized compounds were determined by open glass capillaries using a Polmon melting point apparatus and are uncorrected. Infrared spectra were recorded on Shimadzu FT IR spectrophotometer with KBr. Mass spectra were obtained on VG-7070H mass spectrometer and ${ }^{1} \mathrm{H}$ NMR were recorded at $300 \mathrm{MHz}$ on a Bruker Avance NMR spectrometer in $\mathrm{CDCl}_{3}$ or DMSO- $\mathrm{d}_{6}$ using tetramethyl silane (TMS) as internal standard. Thin layer chromatography was performed on pre-coated silica gel F254 (Merck) and column chromatography was performed using silica gel 60-120 mesh.

\section{Synthesis of 5-amino-3-t-butyl-1-(2', 4'-dinitro)phenyl-1H- pyrazole (3)}

A mixture of 4, 4-dimethyl-3-oxo-pentanenitrile (1, $34 \mathrm{mM})$, 2, 4-dinitrophenyl hydrazine hydrochloride $(2,35 \mathrm{mM})$ and $50 \mathrm{~mL}$ absolute ethanol along with few drops of $\mathrm{AcOH}$ were heated at the reflux temperature for overnight and cooled to room temperature. The mixture was evaporated under vacuum and the residue thus obtained was washed with ether, suspended in EtOAc, and treated with $1 \mathrm{M}$ $\mathrm{NaOH}$ solution. The organic layer then separated, washed with brine, dried over anhydrous magnesium sulphate and concentrated. The solid which separated was collected, then washed with a mixture of ether and hexane to give 5-amino-3-t-butyl-1-(2', 4'-dinitro)phenyl-1H-pyrazole (3) $[22]$.

\section{General procedure for $N$-[3'-t-butyl-1'-(2", 4"-dinitro) phenylpyrazol-5'-yl] benzamide (5a-l)}

To a solution of 5-amino-3-t-butyl-1-(2', 4'-dinitro)phenyl-1Hpyrazole $(3,9.3 \mathrm{mM})$ in dichloromethane $(5 \mathrm{~mL})$, triethyl amine $(23$ $\mathrm{mM}$ ) was added drop wise. The appropriate benzoylchlorides (4a-k, 12 $\mathrm{mM}$ ) in dichloromethane were added to the above reaction mixture drop wise and stirred for 3 hours at room temperature. The mixture was further diluted with dichloromethane and washed with water, brine followed by once again with water. Sodium sulfate was used to dry the organic layer; solvent was removed under vacuum to get the final derivatives (5a-1). All the compounds were purified over silica to get pure $\mathrm{N}$-(3-tert-butyl-2, 4-dinitro-1-phenyl-1H-pyrazol-5-yl) benzamides (5a-1).

\section{Compound characterization}

$\mathrm{N}$-[3'-t-butyl-1'-(2", 4"-dinitro)phenylpyrazol-5'-yl] benzamide 5a: Yield: $85 \%$, m.p.: $190-192^{\circ} \mathrm{C}$, IR (KBr) $v \mathrm{~cm}^{-1}: 3362 \mathrm{~cm}^{-1}$ (NH str.), $3020 \mathrm{~cm}^{-1}$ (CH str. aromatic), $2950 \mathrm{~cm}^{-1}$ (CH str. methyl), $1643 \mathrm{~cm}^{-1}$ (NH bnd) ${ }^{1} \mathrm{H}$ NMR $\left(\mathrm{CDCl}_{3}\right) \delta(\mathrm{ppm}): 1.23$ (s, 9H, $t$-butyl), 6.18 (s, $1 \mathrm{H}, \mathrm{C}_{4}$, pyrazole), 7.15-7.55 (m, 3H, $\mathrm{C}_{3}{ }^{\prime}, \mathrm{C}_{4}{ }^{\prime}, \mathrm{C}_{5}{ }^{\prime}$ aromatic), 7.7-7.8 (m, $2 \mathrm{H}, \mathrm{C}_{5}, \mathrm{C}_{6}$, aromatic), 7.9-8.1 (d, $2 \mathrm{H}, \mathrm{C}_{2}{ }^{\prime}, \mathrm{C}_{6}^{\prime}$, aromatic), $8.2\left(\mathrm{~s}, 1 \mathrm{H}, \mathrm{C}_{3}\right.$, aromatic), 9.61 (bs, $1 \mathrm{H}, \mathrm{NH}$ amide). ESI-MS (m/z): 410, 100\% $[\mathrm{M}+\mathrm{H}]^{+}$.

4-Fluoro-N-[3'-t-butyl-1'-(2", 4"-dinitro)phenylpyrazol-5'-yl] benzamide 5b: Yield: $82 \%$, m.p.: $195-197^{\circ} \mathrm{C}$, IR (KBr) $v \mathrm{~cm}^{-1}: 3200 \mathrm{~cm}^{-1}$ (NH str.), $3018 \mathrm{~cm}^{-1}$ (CH str. aromatic), $2950 \mathrm{~cm}^{-1}$ (CH str. methyl), $1686 \mathrm{~cm}^{-1}$ (CO str.), $1620 \mathrm{~cm}^{-1}$ (NH bnd). ${ }^{1} \mathrm{H} \mathrm{NMR}\left(\mathrm{CDCl}_{3}\right) \delta$ (ppm): 1.35 (s, 9H, t-butyl), 6.21 (s, $1 \mathrm{H}, \mathrm{C}_{4}$, pyrazole), 7.3-7.4 (d, $2 \mathrm{H}, \mathrm{C}_{3}{ }^{\prime}, \mathrm{C}_{5}{ }^{\prime}$ aromatic), $7.55-7.64\left(\mathrm{~d}, 1 \mathrm{H}, \mathrm{C}_{6}\right.$, aromatic), $7.7-8.2\left(\mathrm{~m}, 4 \mathrm{H}, \mathrm{C}_{3}, \mathrm{C}_{5}, \mathrm{C}_{2}{ }^{\prime}\right.$, $\mathrm{C}_{6}^{\prime}$, aromatic), 9.79 (bs, $1 \mathrm{H}, \mathrm{NH}$ amide). ESI-MS (m/z): 428, $100 \%$ $[\mathrm{M}+\mathrm{H}]^{+}$.

3-Fluoro- N-[3'-t-butyl-1'-(2", 4"-dinitro)phenylpyrazol-5'-yl] benzamide 5c: Yield: $80 \%$, m.p.: $191-192^{\circ} \mathrm{C}$, IR $(\mathrm{KBr}) v \mathrm{~cm}^{-1}: 3240 \mathrm{~cm}^{-1}$ 
(NH str.), $3060 \mathrm{~cm}^{-1}$ (CH str. aromatic), $2965 \mathrm{~cm}^{-1}$ (CH str. methyl), $1651 \mathrm{~cm}^{-1}$ (NH bnd). ${ }^{1} \mathrm{H}$ NMR $\left(\mathrm{CDCl}_{3}\right) \delta(\mathrm{ppm}): 1.34$ (s, 9H, t-butyl), $6.32\left(\mathrm{~s}, 1 \mathrm{H}, \mathrm{C}_{4}\right.$, pyrazole), 7.12-7.2 (t, $1 \mathrm{H}, \mathrm{C}_{5}{ }^{\prime}$ aromatic), 7.45-7.57 (d, $1 \mathrm{H}, \mathrm{C}_{4}{ }^{\prime}$ aromatic), $7.62-7.74\left(\mathrm{~d}, 1 \mathrm{H}, \mathrm{C}_{6}\right.$ aromatic), $7.85-8.3(\mathrm{~m}, 4 \mathrm{H}$, $\mathrm{C}_{3}, \mathrm{C}_{5}, \mathrm{C}_{2}{ }^{\prime}, \mathrm{C}_{6}{ }^{\prime}$ aromatic), 9.36 (bs, $1 \mathrm{H}, \mathrm{NH}$ amide). ESI-MS (m/z): 428, $100 \%[\mathrm{M}+\mathrm{H}]^{+}$.

4-Chloro- N-[3'-t-butyl-1'-(2", 4"-dinitro)phenylpyrazol-5'-yl] benzamide 5d: Yield: 70\%, m.p.: 198-200 ${ }^{\circ} \mathrm{C}$, IR (KBr) $v \mathrm{~cm}^{-1}: 3220 \mathrm{~cm}^{-1}$ (NH str.), $3017 \mathrm{~cm}^{-1}$ (CH str. aromatic), $2969 \mathrm{~cm}^{-1}$ (CH str. methyl), $1640 \mathrm{~cm}^{-1}$ (NH bnd). ${ }^{1} \mathrm{H}$ NMR $\left(\mathrm{CDCl}_{3}\right) \delta(\mathrm{ppm}): 1.32$ (s, 9H, $t$-butyl), 6.18 (s, $1 \mathrm{H}, \mathrm{C}_{4}$, pyrazole), 7.2-7.33 (d, $2 \mathrm{H}, \mathrm{C}_{3}{ }^{\prime}, \mathrm{C}_{5}{ }^{\prime}$ aromatic), $7.46-7.55$ (d, $1 \mathrm{H}, \mathrm{C}_{6}$, aromatic), $7.60-8.3$ (m, $4 \mathrm{H}, \mathrm{C}_{3}, \mathrm{C}_{5}, \mathrm{C}_{2}$, $\mathrm{C}_{6}^{\prime}$, aromatic), 9.77 (bs, $1 \mathrm{H}, \mathrm{NH}$ amide). ESI-MS (m/z): 444, 100\% [M+H] ${ }^{+}$.

3-Chloro- N-[3'-t-butyl-1'-(2", 4"-dinitro)phenylpyrazol-5'-yl] benzamide 5e: Yield: 78\%, m.p.: 191-192 ${ }^{\circ} \mathrm{C}$, IR (KBr) $v \mathrm{~cm}^{-1}: 3215 \mathrm{~cm}^{-1}$ (NH str.), $3016 \mathrm{~cm}^{-1}$ (CH str. aromatic), $2975 \mathrm{~cm}^{-1}$ (CH str. methyl), $1653 \mathrm{~cm}^{-1}$ (NH bnd). ${ }^{1} \mathrm{H} \mathrm{NMR}\left(\mathrm{CDCl}_{3}\right) \delta$ (ppm): 1.31 (s, 9H, $t$-butyl), $6.18\left(\mathrm{~s}, 1 \mathrm{H}, \mathrm{C}_{4}\right.$, pyrazole), 7.04-7.1 $\left(\mathrm{t}, 1 \mathrm{H}, \mathrm{C}_{5}{ }^{\prime}\right.$ aromatic $), 7.33-7.41(\mathrm{~d}$, $1 \mathrm{H}, \mathrm{C}_{4}{ }^{\prime}$ aromatic), $7.6-7.67$ (d, $1 \mathrm{H}, \mathrm{C}_{6}$ aromatic), $7.78-8.1\left(\mathrm{~m}, 4 \mathrm{H}, \mathrm{C}_{3}\right.$, $\mathrm{C}_{5}, \mathrm{C}_{2}^{\prime}, \mathrm{C}_{6}^{\prime}$ aromatic), 9.39 (bs, $1 \mathrm{H}, \mathrm{NH}$ amide). ESI-MS (m/z): 444, $100 \%[\mathrm{M}+\mathrm{H}]^{+}$.

4-Methoxy- N-[3'-t-butyl-1'-(2", 4"-dinitro)phenylpyrazol-5'-yl] benzamide 5f: Yield: $73 \%$, m.p.: $197-199^{\circ} \mathrm{C}$, IR $(\mathrm{KBr}) v \mathrm{~cm}^{-1}: 3204 \mathrm{~cm}^{-1}$ (NH str.), $3025 \mathrm{~cm}^{-1}$ (CH str. aromatic), $2968 \mathrm{~cm}^{-1}$ (CH str. methyl), $1635 \mathrm{~cm}^{-1}$ (NH bnd). ${ }^{1} \mathrm{H}$ NMR $(\mathrm{CDCl} 3) \delta(\mathrm{ppm}): 1.25$ (s, 9H, $t$-butyl), 3.4 (s, $3 \mathrm{H}$, methoxy), 6.20 (s, $1 \mathrm{H}, \mathrm{C}_{4}$, pyrazole), 6.9-7.03 (d, $2 \mathrm{H}, \mathrm{C}_{3}^{\prime}, \mathrm{C}_{5}^{\prime}$ aromatic), $7.39-7.47\left(\mathrm{~d}, 2 \mathrm{H}, \mathrm{C}_{6}\right.$, aromatic), $7.8-7.99\left(\mathrm{~m}, 4 \mathrm{H}, \mathrm{C}_{3}, \mathrm{C}_{5}\right.$, $\mathrm{C}_{2}{ }^{\prime}, \mathrm{C}_{6}{ }^{\prime}$, aromatic), 9.36 (bs, $1 \mathrm{H}, \mathrm{NH}$ amide). ESI-MS (m/z): 440, $100 \%$ $[\mathrm{M}+\mathrm{H}]^{+}$.

3-Methoxy- N-[3'-t-butyl-1'-(2", 4"-dinitro)phenylpyrazol-5'-yl] benzamide 5g: Yield: 78\%, m.p.: $197-199^{\circ} \mathrm{C}$, IR $(\mathrm{KBr}) v \mathrm{~cm}^{-1}: 3227 \mathrm{~cm}^{-1}$ (NH str.), $3006 \mathrm{~cm}^{-1}$ (CH str. aromatic), $2972 \mathrm{~cm}^{-1}$ (CH str. methyl), $1615 \mathrm{~cm}^{-1}$ (NH bnd). ${ }^{1} \mathrm{H}$ NMR $\left(\mathrm{CDCl}_{3}\right) \delta$ (ppm): 1.28 (s, 9H, $t$-butyl), 3.7 (s, $3 \mathrm{H}$, methoxy), $6.32\left(\mathrm{~s}, 1 \mathrm{H}, \mathrm{C}_{4}\right.$, pyrazole), 6.87-6.98 (m, $2 \mathrm{H}, \mathrm{C}_{4}^{\prime}$, $\mathrm{C}_{5}{ }^{\prime}$ aromatic), 7.7-7.8 (d, $1 \mathrm{H}, \mathrm{C}_{6}$ aromatic), $7.82-8.05\left(\mathrm{~m}, 4 \mathrm{H}, \mathrm{C}_{3}, \mathrm{C}_{5}\right.$, $\mathrm{C}_{2}{ }^{\prime}, \mathrm{C}_{6}{ }^{\prime}$ aromatic), 9.2 (bs, $1 \mathrm{H}, \mathrm{NH}$ amide). ESI-MS (m/z): 440, $100 \%$ $[\mathrm{M}+\mathrm{H}]^{+}$.

4-Trifluoromethyl- $\quad \mathrm{N}$-[3'-t-butyl-1'-(2", $\quad$ 4"-dinitro) phenylpyrazol-5'-yl] benzamide 5h: Yield: $75 \%$, m.p.: $194-196^{\circ} \mathrm{C}$, IR (KBr) $v \mathrm{~cm}^{-1}: 3210 \mathrm{~cm}^{-1}$ (NH str.), $3005 \mathrm{~cm}^{-1}$ (CH str. aromatic), 2960 $\mathrm{cm}^{-1}$ (CH str. methyl), $1648 \mathrm{~cm}^{-1}$ ( $\mathrm{NH}$ bnd). ${ }^{1} \mathrm{H} \mathrm{NMR}\left(\mathrm{CDCl}_{3}\right) \delta(\mathrm{ppm})$ : 1.36 (s, 9H, t-butyl), 6.25 (s, $1 \mathrm{H}, \mathrm{C}_{4}$, pyrazole), 7.5-7.68 (m, $3 \mathrm{H}, \mathrm{C}_{6}, \mathrm{C}_{3}{ }^{\prime}$, $\mathrm{C}_{5}{ }^{\prime}$ aromatic), $7.87-8.2\left(\mathrm{~m}, 4 \mathrm{H}, \mathrm{C}_{3}, \mathrm{C}_{5}, \mathrm{C}_{2}{ }^{\prime}, \mathrm{C}_{6}{ }^{\prime}\right.$, aromatic), 10.1 (bs, $1 \mathrm{H}$, NH amide). ESI-MS (m/z): 478, 100\% [M+H] $]^{+}$.

4-Methyl- N-[3'-t-butyl-1'-(2", 4"-dinitro)phenylpyrazol-5'-yl] benzamide 5i: Yield: $74 \%$, m.p.: $190-192^{\circ} \mathrm{C}$, IR $(\mathrm{KBr}) v \mathrm{~cm}^{-1}: 3200 \mathrm{~cm}^{-1}$ (NH str.), $3040 \mathrm{~cm}^{-1}$ (CH str. aromatic), $2945 \mathrm{~cm}^{-1}$ (CH str. methyl), $1645 \mathrm{~cm}^{-1}$ (NH bnd). ${ }^{1} \mathrm{H}$ NMR $\left(\mathrm{CDCl}_{3}\right) \delta$ (ppm): 1.34 (s, 9H, $t$-butyl), $2.30\left(\mathrm{~s}, 3 \mathrm{H}, \mathrm{CH}_{3}\right), 6.41\left(\mathrm{~s}, 1 \mathrm{H}, \mathrm{C}_{4}\right.$, pyrazole), 6.74-6.9 (d, $2 \mathrm{H}, \mathrm{C}_{3}{ }^{\prime}, \mathrm{C}_{5}{ }^{\prime}$ aromatic), $7.45-7.54\left(\mathrm{~d}, 1 \mathrm{H}, \mathrm{C}_{6}\right.$, aromatic), $7.76-7.99\left(\mathrm{~m}, 4 \mathrm{H}, \mathrm{C}_{3}, \mathrm{C}_{5}\right.$, $\mathrm{C}_{2}{ }^{\prime}, \mathrm{C}_{6}{ }^{\prime}$, aromatic), 9.68 (bs, $1 \mathrm{H}, \mathrm{NH}$ amide). ESI-MS (m/z): 424, 100\% $[\mathrm{M}+\mathrm{H}]^{+}$.

4-Nitro- N-[3'-t-butyl-1'-(2", 4"-dinitro)phenylpyrazol-5'-yl] benzamide 5j: Yield: $81 \%$, m.p.: $195-197^{\circ} \mathrm{C}$, IR $(\mathrm{KBr}) v \mathrm{~cm}^{-1}: 3243 \mathrm{~cm}^{-1}$ (NH str.), $3028 \mathrm{~cm}^{-1}$ (CH str. aromatic), $2920 \mathrm{~cm}^{-1}$ (CH str. methyl), $1644 \mathrm{~cm}^{-1}$ (NH bnd). ${ }^{1} \mathrm{H} \mathrm{NMR}\left(\mathrm{CDCl}_{3}\right) \delta(\mathrm{ppm}): 1.27$ (s, 9H, $t$-butyl), $6.53\left(\mathrm{~s}, 1 \mathrm{H}, \mathrm{C}_{4}\right.$, pyrazole), 7.6-7.7 (md, $1 \mathrm{H}, \mathrm{C}_{6}$, aromatic), 7.92-8.1 (m, $6 \mathrm{H}, \mathrm{C}_{3}, \mathrm{C}_{5}, \mathrm{C}_{2}{ }^{\prime}, \mathrm{C}_{3}{ }^{\prime}, \mathrm{C}_{5}^{\prime}, \mathrm{C}_{6}{ }^{\prime}$ aromatic), 10.2 (bs, $1 \mathrm{H}, \mathrm{NH}$ amide). ESI-MS $(\mathrm{m} / \mathrm{z}): 455,100 \%[\mathrm{M}+\mathrm{H}]^{+}$.
3-Nitro-N-[3'-t-butyl-1'-(2", 4"-dinitro)phenylpyrazol-5'-yl] benzamide 5k: Yield: $83 \%$, m.p.: $192-195^{\circ} \mathrm{C}$, IR $(\mathrm{KBr}) v \mathrm{~cm}^{-1}: 3240 \mathrm{~cm}^{-1}$ (NH str.), $3028 \mathrm{~cm}^{-1}$ (CH str. aromatic), $2960 \mathrm{~cm}^{-1}$ (CH str. methyl), $1650 \mathrm{~cm}^{-1}$ (NH bnd). ${ }^{1} \mathrm{H}$ NMR $\left(\mathrm{CDCl}_{3}\right) \delta$ (ppm): 1.42 (s, 9H, $t$-butyl), $6.23\left(\mathrm{~s}, 1 \mathrm{H}, \mathrm{C}_{4}\right.$, pyrazole), 7.31-7.4 (t, $1 \mathrm{H}, \mathrm{C}_{5}$ 'aromatic), 7.5-7.64 $(\mathrm{d}, 1 \mathrm{H}$, $\mathrm{C}_{6}$ aromatic), 7.9-8.4 (m, 5H, $\mathrm{C}_{3}, \mathrm{C}_{5}, \mathrm{C}_{2}{ }^{\prime}, \mathrm{C}_{4}^{\prime}, \mathrm{C}_{6}$ ', aromatic), 10.3 (bs, $1 \mathrm{H}$, NH amide). ESI-MS (m/z): 455, 100\% $[\mathrm{M}+\mathrm{H}]^{+}$.

4-Amino- N-[3'-t-butyl-1'-(2", 4"-dinitro)phenylpyrazol-5'-yl] benzamide 5l: Yield: $60 \%$, m.p.: $191-193^{\circ} \mathrm{C}$, IR $(\mathrm{KBr}) v \mathrm{~cm}^{-1}: 3410$ and $3300 \mathrm{~cm}^{-1}$ (NH antisym and sym str.), $3010 \mathrm{~cm}^{-1}$ (CH str. aromatic), $2932 \mathrm{~cm}^{-1}$ (CH str. methyl), $1645 \mathrm{~cm}^{-1}$ (NH bnd). ${ }^{1} \mathrm{H} \mathrm{NMR}\left(\mathrm{CDCl}_{3}\right) \delta$ (ppm): 1.30 (s, 9H, t-butyl), $4.1\left(\mathrm{~s}, 2 \mathrm{H}, \mathrm{NH}_{2}\right), 6.45$ (s, $1 \mathrm{H}, \mathrm{C}_{4}$, pyrazole), 6.8-6.94 (d, $2 \mathrm{H}, \mathrm{C}_{3}{ }^{\prime}, \mathrm{C}_{5}{ }^{\prime}$ aromatic), 7.5-7.58 (d, $1 \mathrm{H}, \mathrm{C}_{6}$, aromatic), 7.8$8.05\left(\mathrm{~m}, 4 \mathrm{H}, \mathrm{C}_{3}, \mathrm{C}_{5}, \mathrm{C}_{2}^{\prime}, \mathrm{C}_{6}^{\prime}\right.$, aromatic), 9.57 (bs, $1 \mathrm{H}, \mathrm{NH}$ amide). ESIMS (m/z): 425, 100\% [M+H] $]^{+}$.

\section{Antibacterial and antifungal activity}

Cultures of Bacillus subtilis (MTCC 441), Staphylocoocus aureus (MTCC 96), Escherichia coli (MTCC 1687), Proteus vulgaris (MTCC 742), Aspergillus flavus (MTCC 870) and Candida albicans (MTCC 854) were used to investigate the antimicrobial activities of compounds $5 \mathrm{a}-1$. The in vitro antimicrobial activity was performed by the serial dilution method to find out minimum inhibitory concentration in Muller Hinton broth. MIC of all the compounds was established according to the guidelines mentioned in National Committee for Clinical Laboratory Standards (NCCLS) document M27-A [23]. DMSO was served as solvent to prepare stock solutions $(100 \mu \mathrm{g} / \mathrm{ml})$ of test compounds and standard drugs, serial dilutions of the compounds $(100,50,25,3.12,1.6,0.8$ and $0.4 \mu \mathrm{g} / \mathrm{ml})$ were prepared from the respective stock solution to determine the MIC employing overnight cultures of all four microbes [24]. MC Farland standards were adopted and Streptomycin and Fluconazole were used as standards. MIC was interpreted as the lowest concentration of the antimicrobial compound which will inhibit the visible growth of the microorganisms after certain period of incubation.

\section{Carrageenan induced rat hind paw edema}

All the newly synthesized compounds 5a-l were screened for antiinflammatory activity in carrageenan induced rat hind paw edema Winter assay method [25]. All tested compounds were suspended in water with few drops of Tween-80. Fourteen groups of 6 wistar rats weighing 150-200 g were grouped for test, standard and control. The animals were housed in standard conditions with food and water ad libitum. A single dose of $10 \mathrm{mg} / \mathrm{Kg}$ of compounds $5 \mathrm{a}-\mathrm{l}$ was administered orally to the respective groups and after 30 minutes carrageenan (1\%, 1 $\mathrm{mL}$ was injected intradermally into the intraplantar region of the right hind paw) was injected in plantar region of hind paw for all groups. The control group received $1 \mathrm{~mL}$ vehicle and Diclofenac $50 \mathrm{mg} / \mathrm{Kg}$ dose was administered in control and standard group respectively. Rat edema was evaluated by measuring the rat paw volume at intervals 1, 2, 3 and 4 hour using digital plethysmometer (Ugo Basile) [26]. The inhibition percentage was calculated as:

\section{$\%$ Inhibition of edema $=(1-\mathrm{Et} / \mathrm{Em}) \times 100$}

Where Et $(\mathrm{mL})$ represents the average value of the edema in $\mathrm{ml}$ in treated groups in 1-4 h after carrageenan injection, while Em $(\mathrm{mL})$ represents the average value of the edema in $\mathrm{ml}$ in control group in 1-4 $\mathrm{h}$ after carrageenan injection. 


\section{Results and Discussion}

\section{Chemistry}

In our previous studies, we synthesized several amide derivatives and tested them for their anti-inflammatory activity [27]. As part of our continuous efforts in this area, a series of some new pyrazole derivatives containing substituent's at 1,3,5-positions were synthesized according to Scheme 1. The intermediate compound 5-amino-3-t-butyl-1-(2', 4'-dinitro)phenyl-1H-pyrazole 3 was prepared in good yields by refluxing 4, 4-dimethyl-3-oxo-pentanenitrile 1 and 2, 4-dinitrophenyl hydrazine hydrochloride 2 . The FT IR spectrum of 3 showed the presence of bands characteristic for primary amine at $3423.42 \mathrm{~cm}^{-1}$ and $3264.21 \mathrm{~cm}^{-1}$ which are attributed to the asymmetric and symmetric stretching respectively and aromatic hydrogen stretching band located at $3056.75 \mathrm{~cm}^{-1}$. The $\mathrm{CH}_{3}$ groups of t-butyl vibrations were observed at $2961.33 \mathrm{~cm}^{-1}$ and $2828.42 \mathrm{~cm}^{-1}$ and peak assigned for bending vibration of $\mathrm{NH}$ group was observed at $1625.94 \mathrm{~cm}^{-1}$. The ${ }^{1} \mathrm{H}$ NMR of 3 revealed a broad singlet at $\delta 3.77 \mathrm{ppm}$ characteristic for primary amine group, multiplet at delta value of $8.05,7.35$ and 7.63 for $\mathrm{C}_{3}, \mathrm{C}_{5}$ and $\mathrm{C}_{6}$ aromatic protons and a pyrazolyl- $\mathrm{C}_{4}-\mathrm{H}$ as a singlet at $6.1 \mathrm{ppm}$. The nine $t$-butyl protons were found as singlet at $\delta 1.23 \mathrm{ppm}$. The EI Mass spectrum of 3 showed molecular ion peak at $\mathrm{m} / \mathrm{z} 215$.

When 5-amino-3-t-butyl-1-(2', 4'-dinitro)phenyl-1H-pyrazole 3 was stirred with substituted benzoylchlorides $4 \mathrm{a}-\mathrm{k}$ in dichloromethane and triethylamine, pyrazolylbenzamides 5a-l were obtained in moderate to good yields ( $5 \mathrm{j}$ was reduced with stannous chloride to form 5l). The structures of the isolated compounds were determined by spectral methods.
The FT IR spectrum of 5 a revealed characteristic NH band at 3362 $\mathrm{cm}^{-1}$, the aromatic hydrogen stretching was found at $3020 \mathrm{~cm}^{-1}$ and stretching vibrations of $\mathrm{CH}_{3}$ group of $t$-butyl band was noticed at 2950 $\mathrm{cm}^{-1}$. A band at $1595 \mathrm{~cm}^{-1}$ was assigned for the $\mathrm{C}=\mathrm{C}$ stretching. The ${ }^{1} \mathrm{H}$ NMR spectra displayed a broad absorption peak at $\delta 9.61$ which was due to resonance of $\mathrm{NH}$ proton of amide while the pyrazol- $\mathrm{C}_{4}-\mathrm{H}$ and nine t-butyl protons appears as singlet's at $\delta 6.18$ and $1.23 \mathrm{ppm}$ respectively. The aromatic protons appeared as doublet at $\delta$ 7.9-8.1 which indicated the resonance of $\mathrm{C}_{2}{ }^{\prime}, \mathrm{C}_{6}{ }_{6}$ protons. Three aromatic protons $\mathrm{C}_{3}{ }^{\prime}, \mathrm{C}_{4}{ }^{\prime}, \mathrm{C}_{5}{ }^{\prime}$ resonated as multiplet at $\delta$ 7.15-7.55 and another multiplet at $\delta$ 7.7-7.8 integrated for two protons $\mathrm{C}_{5}$ and $\mathrm{C}_{6} . \mathrm{C}_{3}$ proton was observed as singlet at $\delta 8.2$.

\section{Pharmacology}

Antimicrobial activity: All synthesized compounds 5 (a-l) were screened for in vitro activity against Bacillus subtilis, Staphylococcus aureus representing Gram positive bacteria, E. coli, Proteus vulgaris representing Gram negative organism, Aspergillus flavus and Candida albicans representing fungal strains. Streptomycin and Fluconazole were used as antibacterial and antifungal standard compound respectively during the studies. The antimicrobial activity of the synthesized compound was evaluated by measuring the MIC value and their results were compared with those of standards and are represented in Tables 1 and 2.

All the N-[3'-t-butyl-1'-(2", 4"-dinitro)phenylpyrazol-5'-yl] benzamides (5a-l) displayed antibacterial activity in the range of 3.12 - $100 \mu \mathrm{g} / \mathrm{mL}$ concentrations. Among the tested compounds, it was noticed that compounds $5 \mathrm{a}$ demonstrated MIC value of $50 \mu \mathrm{g} / \mathrm{mL}$ against Gram positive bacteria and $100 \mu \mathrm{g} / \mathrm{mL}$ against Gram negative

\begin{tabular}{|c|c|c|c|c|c|c|}
\hline Comp No & Mol. For. & Mol. Wt. & Yield (\%) & m.p. $\left({ }^{\circ} \mathrm{C}\right)$ & $\mathbf{R}_{\mathrm{f}}$ & CLogP \\
\hline $5 a$ & $\mathrm{C}_{20} \mathrm{H}_{19} \mathrm{~N}_{5} \mathrm{O}_{5}$ & 409.40 & 85 & 190-192 & 0.49 & 4.44 \\
\hline $5 b$ & $\mathrm{C}_{20} \mathrm{H}_{18} \mathrm{FN}_{5} \mathrm{O}_{5}$ & 427.39 & 82 & 195-197 & 0.48 & 4.60 \\
\hline $5 c$ & $\mathrm{C}_{20} \mathrm{H}_{18} \mathrm{FN}_{5} \mathrm{O}_{5}$ & 427.39 & 80 & 191-192 & 0.50 & 4.60 \\
\hline $5 d$ & $\mathrm{C}_{20} \mathrm{H}_{18} \mathrm{ClN}_{5} \mathrm{O}_{5}$ & 443.84 & 70 & $198-200$ & 0.52 & 5.17 \\
\hline $5 e$ & $\mathrm{C}_{20} \mathrm{H}_{18} \mathrm{CIN}_{5} \mathrm{O}_{5}$ & 443.84 & 78 & 191-192 & 0.55 & 5.17 \\
\hline $5 f$ & $\mathrm{C}_{21} \mathrm{H}_{21} \mathrm{~N}_{5} \mathrm{O}_{6}$ & 439.42 & 73 & 197-199 & 0.53 & 4.53 \\
\hline $5 g$ & $\mathrm{C}_{21} \mathrm{H}_{21} \mathrm{~N}_{5} \mathrm{O}_{6}$ & 439.42 & 78 & 197-199 & 0.54 & 4.53 \\
\hline $5 \mathrm{~h}$ & $\mathrm{C}_{21} \mathrm{H}_{18} \mathrm{~F}_{3} \mathrm{~N}_{5} \mathrm{O}_{5}$ & 477.39 & 75 & $194-196$ & 0.51 & 5.36 \\
\hline $5 i$ & $\mathrm{C}_{21} \mathrm{H}_{21} \mathrm{~N}_{5} \mathrm{O}_{5}$ & 423.42 & 59 & $233-236$ & 0.56 & 4.25 \\
\hline $5 j$ & $\mathrm{C}_{20} \mathrm{H}_{18} \mathrm{~N}_{6} \mathrm{O}_{7}$ & 454.39 & 81 & $195-197$ & 0.45 & 4.34 \\
\hline $5 k$ & $\mathrm{C}_{20} \mathrm{H}_{18} \mathrm{~N}_{6} \mathrm{O}_{7}$ & 454.39 & 83 & 192-195 & 0.61 & 4.34 \\
\hline 51 & $\mathrm{C}_{20} \mathrm{H}_{20} \mathrm{~N}_{6} \mathrm{O}_{5}$ & 424.41 & 60 & 191-193 & 0.65 & 3.53 \\
\hline
\end{tabular}

Table 1: Physical properties of synthesized compounds.

\begin{tabular}{|c|c|c|c|c|c|c|c|c|}
\hline \multirow[t]{2}{*}{ S No } & \multirow[t]{2}{*}{ Comp Code } & \multirow[t]{2}{*}{$\mathbf{R}$} & \multicolumn{4}{|c|}{ Anti-bacterial } & \multicolumn{2}{|c|}{ Anti-fungal } \\
\hline & & & BS & SA & EC & PV & AF & CA \\
\hline & $5 a$ & $\mathrm{H}$ & 50 & 50 & 100 & 100 & 12.5 & 50 \\
\hline & $5 b$ & $4-\mathrm{F}$ & 6.25 & 12.5 & 6.25 & 6.25 & 3.12 & 6.25 \\
\hline & $5 c$ & $3-\mathrm{F}$ & 100 & 100 & 50 & 50 & 100 & 100 \\
\hline & $5 d$ & $4-\mathrm{Cl}$ & 12.5 & 12.5 & 12.5 & 12.5 & 3.12 & 6.25 \\
\hline & $5 e$ & $3-\mathrm{Cl}$ & 100 & 100 & 50 & 50 & 12.5 & 50 \\
\hline & $5 f$ & $4-\mathrm{OCH}_{3}$ & 3.12 & 3.12 & 12.5 & 6.25 & 3.12 & 3.12 \\
\hline & $5 g$ & $3-\mathrm{OCH}_{3}$ & 100 & 100 & 50 & 50 & 50 & 12.5 \\
\hline & $5 \mathrm{~h}$ & $4-\mathrm{CF}_{3}$ & 3.12 & 3.12 & 6.25 & 6.25 & 3.12 & 3.12 \\
\hline & $5 i$ & $4-\mathrm{CH}_{3}$ & 6.25 & 6.25 & 12.5 & 12.5 & 6.25 & 6.25 \\
\hline & $5 j$ & $4-\mathrm{NO}_{2}$ & 3.12 & 3.12 & 12.5 & 12.5 & 6.25 & 6.25 \\
\hline & $5 \mathrm{k}$ & $3-\mathrm{NO}_{2}$ & 100 & 100 & 50 & 50 & 12.5 & 12.5 \\
\hline & 51 & 4- $\mathrm{NH}_{2}$ & 50 & 50 & 50 & 50 & 100 & 100 \\
\hline \multicolumn{3}{|c|}{ Streptomycin } & 50 & 50 & 50 & 50 & & \\
\hline \multicolumn{3}{|c|}{ Fluconazole } & & & & & 50 & 50 \\
\hline
\end{tabular}

Table 2: Antimicrobial activity (MIC in microgram $/ \mathrm{mL}$ ) of compounds 5a-l. 
Citation: Fatima A, Kulkarni R, Mantipragada B (2015) Design, Synthesis, Antimicrobial and Anti-inflammatory Activity of N-Pyrazolyl Benzamide Derivatives. Med chem 5: 521-527. doi: 10.4172/2161-0444.1000311

\begin{tabular}{|c|c|c|c|c|c|c|}
\hline \multirow[t]{2}{*}{ S No } & \multirow[t]{2}{*}{ Comp Code } & \multirow[t]{2}{*}{$\mathbf{R}$} & \multicolumn{4}{|c|}{$\%$ inhibition at different intervals } \\
\hline & & & $1 \mathrm{hr}$ & $2 \mathrm{hr}$ & $3 \mathrm{hr}$ & $4 \mathrm{hr}$ \\
\hline & $5 a$ & $\mathrm{H}$ & 51.4 & 55.7 & 71.3 & 75.6 \\
\hline & $5 b$ & $4-\mathrm{F}$ & 49.6 & 51.4 & 63.1 & 61.9 \\
\hline & $5 c$ & $3-\mathrm{F}$ & 40.3 & 54.7 & 60.2 & 56.1 \\
\hline & $5 d$ & 4- $\mathrm{Cl}$ & 57.6 & 72.5 & 77.9 & 87.6 \\
\hline & $5 e$ & $3-\mathrm{Cl}$ & 66.3 & 74.1 & 77.8 & 53.4 \\
\hline & $5 f$ & $4-\mathrm{OCH}_{3}$ & 64.9 & 73.8 & 78.9 & 83.6 \\
\hline & $5 g$ & $3-\mathrm{OCH}_{3}$ & 51.3 & 60.1 & 65.2 & 51.7 \\
\hline & $5 \mathrm{~h}$ & $4-\mathrm{CF}_{3}$ & 65.4 & 86.5 & 87.9 & 89.4 \\
\hline & $5 i$ & $4-\mathrm{CH}_{3}$ & 58.3 & 70.1 & 75.2 & 82.2 \\
\hline & $5 j$ & $4-\mathrm{NO}_{2}$ & 69.4 & 78.3 & 81.1 & 83.2 \\
\hline & $5 \mathrm{k}$ & $3-\mathrm{NO}_{2}$ & 62.5 & 76.8 & 79.1 & 80.3 \\
\hline & 51 & $4-\mathrm{NH}_{2}$ & 43.6 & 55.1 & 60.2 & 62.2 \\
\hline \multicolumn{3}{|c|}{ Diclofenac (50 mg/Kg) } & 54 & 77.9 & 82.1 & 92.3 \\
\hline
\end{tabular}

Table 3: Anti-inflammatory activity (\% inhibition) of compounds $5 a-1$.<smiles>CC(C)(C)C(=O)CC#[I-]</smiles>

1

$\mathbf{R}=$

5a-l

$\mathrm{a}=\mathrm{H}$

$\mathrm{b}=4 \mathrm{~F}$

$\mathrm{c}=3 \mathrm{~F}$

$\mathrm{d}=4 \mathrm{Cl}$

$\mathrm{e}=3 \mathrm{Cl}$

$\mathrm{f}=4 \mathrm{OMe}$

$\mathrm{g}=3 \mathrm{OMe}$

$\mathrm{h}=4 \mathrm{CF}_{3}$

$\mathrm{i}=4 \mathrm{Me}$

$\mathrm{j}=4 \mathrm{NO}_{2}$

$\mathrm{k}=3 \mathrm{NO}_{2}$

$5 \mathbf{j}$ is reduced to $5 \mathbf{l}$

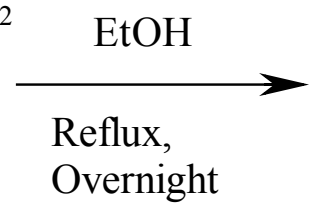

2<smiles>[R]c1cccc(C(=O)Cl)c1</smiles>

3

DCM,

TEA

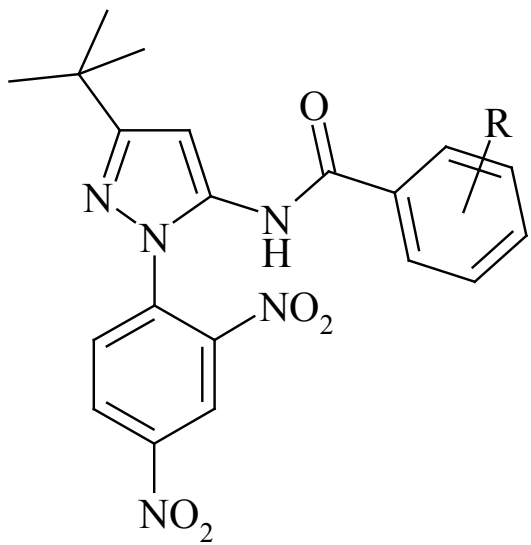

5a-l 
bacteria. Compounds $5 \mathrm{f}, 5 \mathrm{~h}, 5 \mathrm{j}$ have shown higher activity than standard, while the compounds $5 \mathrm{c}, 5 \mathrm{e}, 5 \mathrm{~g}$ and $5 \mathrm{k}$ have shown lesser activity against both Gram positive and Gram negative bacteria. Rests of the molecules were active in the range of $6.25-50 \mu \mathrm{g} / \mathrm{mL}$. The most effective compounds $5 \mathrm{f}, 5 \mathrm{~h}, 5 \mathrm{j}$ with MIC value of $3.12 \mu \mathrm{g} / \mathrm{mL}$ were found to be potent against both the gram Gram positive organisms.

All the compounds were also evaluated for their antifungal activity against Aspergillus flavus and Candida albicans and results were compared against Fluconazole as standard. Compounds 5a-1 demonstrated antifungal activity against both the tested organisms with MIC values in the range of $3.12-50 \mu \mathrm{g} / \mathrm{mL}$. Among the compounds, $5 \mathrm{f}$ and $5 \mathrm{~h}$ were most potent against fungal strains with MIC value of $3.12 \mu \mathrm{g} / \mathrm{mL}$ followed by compounds $5 \mathrm{i}$ and $5 \mathrm{j}$ with $6.25 \mu \mathrm{g} / \mathrm{mL}$ against both Aspergillus flavus and Candida albicans as comparable to that of Fluconazole. Compounds $5 \mathrm{~b}$ and $5 \mathrm{~d}$ were highly active against only one strain and displayed promising antifungal activity at MIC $3.12 \mu \mathrm{g} / \mathrm{mL}$. In general, the target compounds $5 \mathrm{f}, 5 \mathrm{~h}$ and $5 \mathrm{j}$ with substitution like methoxy, trifluoromethyl, nitro at para position respectively showed more significant antimicrobial activity than standard. Two compounds $5 \mathrm{c}$ and 51 were least potent with MIC $100 \mu \mathrm{g} / \mathrm{mL}$.

Anti-inflammatory activity: All the twelve synthesized molecules 5a-l were further explored for anti-inflammatory activity by rat paw induced edema model and the results were compared against blank and reference as Diclofenac, the results are listed down in the Table 3. A dose of $10 \mathrm{mg} / \mathrm{Kg}$ was set for all test compounds which were administered orally as suspension. Almost all of the tested compounds showed promising anti-inflammatory activity at $4^{\text {th }}$ hour of carrageenan administration however four compounds $5 \mathrm{~b}, 5 \mathrm{c}, 5 \mathrm{e}$ and $5 \mathrm{~g}$ showed decrease in anti-inflammatory activity at the $4^{\text {th }}$ hour. Compounds $5 \mathrm{c}$, $5 \mathrm{e}$ and $5 \mathrm{~g}$ demonstrated $50 \%$ anti-inflammatory activity at $4^{\text {th }}$ hour and this may be attributed to the substitution at meta or $3^{\text {rd }}$ position with chloro, methoxy and nitro groups. Unsubstituted compound $5 \mathrm{a}$ displayed significant activity of $75.6 \%$ at the $4^{\text {th }}$ hour.

Among the synthesized compounds highest potency was exhibited by compound $5 \mathrm{~h}$ in comparison to Diclofenac with the activity of $89.4 \%$ inhibition followed by compound $5 \mathrm{~d}, 5 \mathrm{f}, 5 \mathrm{j}, 5 \mathrm{i}$ and $5 \mathrm{k}$ which demonstrated over $80 \%$ activity at $4^{\text {th }}$ hour. The activity was found to be increasing when electron withdrawing groups were inserted at $R_{4}$ at para position among them only $5 \mathrm{~b}$ and $5 \mathrm{l}$ with fluoro and amino substituent showed less activity.

\section{Conclusion}

Various N-[3'-t-butyl-1'-(2", 4"-dinitro)phenylpyrazol-5'-yl] benzamides $(5 \mathrm{a}-\mathrm{l})$ were synthesized by following reported methods and the structure of the compounds 5 a-l were confirmed by various analytical techniques. The target compounds (5a-l) were evaluated for antimicrobial and anti-inflammatory activities and showed potent antimicrobial, anti-inflammatory activity.

Amongst the compounds screened, the highest potent activity was observed for compound $5 \mathrm{~h}$ for both anti-inflammatory activity and antimicrobial activity. Several compounds exhibited peak anti-inflammatory activity in the $3^{\text {rd }}$ and $4^{\text {th }}$ hour of carrageenan administration. Compounds 5d, 5f, 5h, 5i, 5j and 5k showed over $80 \%$ anti-inflammatory activity even in the $4^{\text {th }}$ hour which may be due to presence of substitution at para position and among the entire compounds screened compound $5 \mathrm{~h}$ was found to be the most potent compound.

Antimicrobial activity of compounds for revealed that maximum compounds showed MIC value lesser than $50 \mu \mathrm{g} / \mathrm{mL}$ and compounds $5 \mathrm{f}, 5 \mathrm{~h}$ and $5 \mathrm{j}$ showed MIC value $3.12 \mu \mathrm{g} / \mathrm{mL}$ against numerous strains.
Compounds $5 \mathrm{f}$ and $5 \mathrm{~h}$ also exhibited MIC value $3.12 \mu \mathrm{g} / \mathrm{mL}$ against both fungal strains and compound $5 \mathrm{~d}$ with para chloro substitution has MIC value $3.12 \mu \mathrm{g} / \mathrm{mL}$ against one fungal strain. This study will definitely beneficial to develop future anti-inflammatory and antimicrobial compounds with potent activity.

\section{References}

1. Almekinders LC, Gilbert JA (1986) Healing of experimental muscle strains and the effects of nonsteroidal antiinflammatory medication. Am J Sports Med 14: 303-308.

2. Umare V, Pradhan V, Nadkar M, Rajadhyaksha A, Patwardhan M, et al. (2014) Effect of Proinflammatory Cytokines (IL-6, TNF-a, and IL-1ß) on Clinical Manifestations in Indian SLE Patients. Mediators Inflamm 4: 253-261.

3. Baechler EC, Batliwalla FM, Karypis G, Gaffney PM, Ortmann WA, et al. (2003) Interferon-inducible gene expression signature in peripheral blood cells of patients with severe lupus. Proc Natl Acad Sci USA 100: 2610-2615.

4. Khazi IM, Koti RS, Gadad AK, Mahajanshetty CS, Shivakumar YS, et al. (2004) Synthesis of biologically active benzothiazole derivatives. Ind J Chem 43B: 393-396.

5. Karbasanagouda T, Adhikari AV, Shetty NS (2007) A new approach for the synthesis of some novel sulfur bridged pyrazoles and their characterization. Euro J med Chem 42: 521.

6. Desalegn A (2014) Antimicrobial activity of medicinal plant extracts and their synergistic effect on some selected pathogens. Am J Ethnomedicine 1 Suppl 1: 18-29.

7. Pattan SR, Desai NS, Rabara PA, Bukitgar AA, Wakale VS (2008) Synthesis of substituted fluoro pyrazoles for anti-bacterial and anti-oxidant activity. Ind J Pharmac Educ and Res 42 Suppl 4: 314-318.

8. David ML, Blasser M, Carrs O (2011) Discovery research the scientific challenge of finding new antibiotics. J Antimicr Chemother 66 Suppl 8: 2202-2208.

9. Borathakur SK, Boruah P, Goswami BN (2007) [4+2] Diels-Alder cycloaddition reaction of 2-benylidineamino-4-phenyl-1,3-thiazoles with sulfene and their antifungal activities. J ChemRes 12: 127-132.

10. Murad S, Pinnel SR, Alexandria (1998) Synthesis, anti-inflammatory and ulcerogenicity studies of novel substituted and fused pyrazolopyrimidines. Sci Exch 7: 33-36.

11. Wood JD, Gorecki DK, Dimmock JR, Hawes EM (1973) Molecular StructureActivity Relationships of Hydrazides Inhibiting Glutamic Acid Decarboxylase

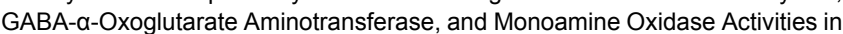
Chick Brain. Can J Physiol Pharmacol 53: 47-55.

12. van Hal SJ, Barbagiannakos T, Jones M, Wehrhahn MC, Mercer J, et al. (2011) Methicillin resistant Staphylococcus aureus Vancomycin susceptibility testing methodology correlations, temporal trends and clonal patterns. J Antimicr Chemother 66 Suppl 10: 2284-2287.

13. Pattan SR, Dighe NS, Nirmal SA, Merekar AN, Laware RB, et al. (2009) Synthesis and biological evaluation of some substituted amino thiazole derivatives. Asian J Res Chem 2: 196-201.

14. Vipin M, Vashishtha (2010) Growing Antibiotics Resistance and the Need for New Antibiotics. Ind Pediat 47: 505-506.

15. Moged AB, Evelin BM (2003) Convergent synthesis and antibacterial activity of pyrazole and pyrazoline derivatives of diazepam. Eur J Pharm Sci 20 Suppl 2: $173-179$.

16. Quiroga J, Rodrigo A, Manuel N, Sanchez A, Justo C (2010) Synthesis of novel pyrazolic analogues of chalcones and their 3-aryl-4-(3-aryl-4,5-dihydro- $1 \mathrm{H}$ pyrazol-5-yl)-1-phenyl-1H-pyrazole derivatives as potential antitumor agents. Bioorg Med Chem 18: 4965-4974.

17. Shin-Ru S, Tzu-Yun C, Gadarla Randheer R, Sung-Nain T, Hsiun-Ling C, et al. (2010) Peyserarachzole compound BPR1P0034 with potent and selective anti-influenza virus activity. J of Biomed Sci 17 Suppl 13: 1-9.

18. Livermore DM (2004) The need for new antibiotics. Clin Microbiol Infect 10 Suppl 4: 1-9

19. Dumas J, Hatoum-Mokdad H, Sibley RN, Smith RA, Scott WJ, et al. (2002) Synthesis and pharmacological characterization of a potent, orally active p38 kinase inhibitor. Bioorg Med Chem Lett 12: 1559-1562. 
Citation: Fatima A, Kulkarni R, Mantipragada B (2015) Design, Synthesis, Antimicrobial and Anti-inflammatory Activity of N-Pyrazolyl Benzamide Derivatives. Med chem 5: 521-527. doi: 10.4172/2161-0444.1000311

20. Priyadarsini P, Ujwalaa B, Venkata Rao B, Madhava Rao V (2012) Synthesis and antimicrobial activity of some novel pyrazoles. Der Pharm Lett 4 Suppl 4 : 1123-1128.

21. Bhandari SV, Bothara KG, Raut MK, Patil AA, Sarkate AP (2008) Design, synthesis and evaluation of anti-inflammatory, analgesic studies of novel s-substituted phenacyl -, 3, 4-thiadiazole and Schiff bases of diclofenac acid as nonulcerogenic derivatives. Bioorg Med Chem 16: 1822-1831.

22. Kulkarni R, Vijay K, Harish B, Krishna K, Naveen G, et al. (2014) Synthesis, antibacterial, antifungal and antitubercular activities of $\mathrm{N}$-pyrazolylbenzamide derivatives. Med Chem 10: 220-227.

23. NCCLS (1997) National Committee for Clinical Laboratory Standards (NCCLS) Approved standard M27 Wayne A, PA, USA.
24. Andrews JM (2001) Determination of minimum inhibitory concentrations. J Antimicrob Chemother 48 Suppl 1: 5-16.

25. Winter CA, Risley EA, Nuss GW (1962) Carrageenin-induced edema in hind paw of the rat as an assay for antiiflammatory drugs. Proc Soc Exp Biol Med 111: 544-547.

26. Still WC, Tempczyk A, Hawley RC, Hendrickson T (1990) Semi analytical treatment of solvation for molecular mechanics and dynamics. J Am Chem Soc 112: 6127-6129.

27. Aneesa F, Kulkarni R, Bhagavanraju M (2015) Design and synthesis of $\mathrm{N}$-substituted amino thiazole compounds as anti-inflammatory agents. Der Pharm Chem 7: 212-220. 